\title{
Improvement of the cardiopulmonary capacity of patients with chronic obstructive pulmonary disease of Kinshasa by training of aerobic endurance and resistance
}

\author{
Mabele Godefroid Kusuayi ${ }^{1}$, Ekisawa Constant Nkiama ${ }^{1}$, Ngasa Nicias Kiana ${ }^{1}$, \\ Nzeloka Jolie Bongo ${ }^{1}$, Ntumba Jean Marie Kayembe ${ }^{2}$
}

\author{
${ }^{1}$ Laboratory of Medical Fitness and Functional Exercises, Department of Kinesiology, Department of Physical Medicine and \\ Rehabilitation, Faculty of Medicine, University of Kinshasa, Democratic Republic of Congo. \\ ${ }^{2}$ Department of Internal Medicine, Department of Pneumology Faculty of Medicine, University of Kinshasa, Democratic Republic \\ of Congo.
}

\begin{abstract}
The objective is to determine the effect of aerobic endurance training and resistance on cardiorespiratory fitness in patients with Chronic Obstructive Pulmonary Disease in Kinshasa, Democratic Republic of Congo. In a follow-up study with a duration of 36 weeks, between March and May 2018, 30 mismatched patients with a mean age of $49 \pm 9.7$ years followed after medical management at the Kinesiology Department's medical fitness laboratory and functional exercises of University Clinics of Kinshasa, were subject to an aerobic endurance and resistance training program of 3 days per week, for one hour 30 minutes per day, three sets of 20 minutes of work and 10 minutes of rest, of moderate intensity including: treadmill walking, ergo-cycle exercises with progressive loading and respiratory gymnastics. The paired Student $\mathrm{T}$ test was used to compare the continuous variables before and after the program. A significant decrease in most of the parameters studied respectively: $(-23 \mathrm{bpm}) \mathrm{p}<0.0001$ for resting heart rate; (- $26 \mathrm{bpm}) ; \mathrm{p}<0.0001$ for heart rate immediately after exercise $(-10 \mathrm{~mm} \mathrm{Hg}) ; \mathrm{p}<0.0001$ for systolic blood pressure; (- 8 cycles per minute); $\mathrm{p}<0.0001$ for the respiratory rate; ($20.7 \%) ; p<0.0001$ for workload (intensity of effort) and (- 10 minutes); $\mathrm{p}<0.0001$ for dyspnea on exertion. On the other hand, a significant increase of: (+ 2.6 liter per second); $p$ $<0.0001$ for Peak Expiratory Flow; $(+19 \%) ; \mathrm{p}<0.0001$ for the Tiffeneau index; ( +51 minutes); $\mathrm{p}<0.0001$ for the duration of sensation of fatigue during exercise; $(+6 \%), p<0.0001$ for oxygen saturation in the open air and (+ 640 meters); $\mathrm{p}$ $<0.0001$ for the walking area. A training program for aerobic endurance and resistance significantly improves the stress tolerance of patients with Chronic Obstructive Pulmonary Disease and can be considered as an effective
\end{abstract}

non-pharmacological management for the improvement of physical capacity as well as the quality of life of these patients.

Keywords. Aerobic endurance, cardiorespiratory capacity, MPCO, training.

\section{Introduction}

hronic Obstructive Pulmonary Disease
(COPD) mainly brings together two
conditions, chronic bronchitis and emphysema, and may also include a third in the form of chronic asthma (Gauthier et al., 2002; O'Donnell et al., 2003). These three diseases have different pathogenesis mechanisms, but can all lead to chronic obstruction of respiratory flow (Pauwls et al., 2001). COPD is the fourth leading cause of death in industrialized countries and remains the only one of the five leading causes of death that has increased in recent years; there is an increase in the number of deaths of $68 \%$ between 1978 and 1998 (Bryanto et al., 2001; NCHS, 2003). Despite this, as with prevalence data, mortality statistics should be interpreted with caution. In many cases, co-morbidity and diseaserelated complications are reported as causes of death, leading to a significant underestimation of the COPD mortality rate (Bryanto et al., 2001; Camilli et al., 1991; Walk et al., 2003). At the international level, COPD continues to progress in the list of most 
prevalent and life-threatening diseases. Airway obstruction results in reduced expiratory flow rates, such as FEV1, FVC (forced vital capacity) and the ratio of these two rates, the tiffeneau index (FEV1 / FVC) (Pauwels et al., 2001). The characteristic parenchymal destruction of emphysema causes a decrease in elastic recoil and a collapse of the respiratory tract at exhalation, which increases the pulmonary volumes at rest, a phenomenon which is accentuated during an effort. This inability of the lung to empty normally causes a decrease in physical performance by increasing functional residual capacity and total lung capacity and pushes the individual's tidal volume more and more towards maximal volumes. Since dyspnea is the main complaint of patients with COPD, the various treatments offered will try to act, directly or indirectly, on its multiple causes. A physical exercise program of aerobic endurance and rehabilitative resistance is an important component of COPD treatment (American Thoracic, 1999; Maltais et al., 1996; La casse et al., 1996; Fahy et al., 2004). Its effects on quality of life make it an integral part of the treatment of COPD patients (Ries et al., 1997). Several meta-analyzes show that a program of physical exercises of aerobic endurance and resistance increases exercise tolerance, decreases dyspnea and fatigue, and increases the feeling of control over the disease (Salman et al., 2003; Ambach et al., 1999; Kusuayi et al., 2018b). Since very little intervention can improve lung function in patients with COPD, the benefits in terms of quality of life are an important measure of treatment efficacy (Tomas et al., 2004). This disease therefore has drastic consequences on physical fitness and it is important to be concerned about it. In the Democratic Republic of Congo, the effect of aerobic endurance training and resistance on the physical condition of patients with Chronic Obstructive Pulmonary Disease is not very well known. It is to fill this gap that the present work has been undertaken.

\section{Methods}

\section{Participants}

This is an experimental follow-up study, of 30 deconditioned patients with a mean age of $49 \pm 9.7$ years, with dyspnea and low-effort fatigue were submitted for 36 weeks, between March and May 2018 followed at the Medical Fitness Laboratory and Functional Kinesiology Service Exercises of University Clinics of Kinshasa. Physical deconditioning and exercise intolerance were characterized by a percentage increase in maximum frequency, dyspnea, fatigue with less effort, and a decrease in the ratio of maximal volume exhaled in the first second (FEV1) / vital capacity (CVF) (Tiffeneau index, TIFF), a decrease in peak expiratory start (PEF) and a reduction in mobility (perimeter of walking). The inclusion criterion included being followed in the pneumology department of the Kinshasa university clinics for chronic bronchitis, emphysema and / or chronic asthma. All participants were free of consequences and provided informed consent before participating in the study program. The study was conducted in accordance with the Helsinki Declaration and approved by the Institutional Review Board of Kinshasa University Clinic prior to registration of participants.

\section{Exercise protocol}

An aerobic endurance and resistance training program of 3 days per week, for one hour per day, of moderate intensity including a session of continuous respiratory gymnastics for 10 minutes and on the motorized treadmill: Walk of $0,5 \mathrm{~km} 30$ minutes. Charge (prescribed individually according to the patient's fitness). No inclination. No charge at the first session; or ergo-meter cycle: pedaling (50 RPM). Charge (prescribed individually according to the patient's fitness). No charge at the first session. A warm-up of 5 minutes before exercise sessions and cardiovascular training on the seated patient realizing gentle joint movements of neck, shoulder, arms, waist, knees and feet (rotation, flexion and extension). of the body (10 minutes) with light weight exercises, individually prescribed depending on the physical form of the patient (biceps flexion and supine arm: elbow flexion-extension, counter gravity, bringing hand to the shoulder (2 series, 10 repetitions), shoulder press: Shoulder flexionextension, counter gravity, lifting the right arm (2 sets, 10 repetitions), side elevation: arms raised across the movement arch, abduction-adduction ( 2 series, 10 repetitions), anterior deltoid frontal elevation: arms raised at the front of the body flexionshoulder extension ( 2 series, 10 repetitions) and a rest of 5 minutes including the control of the blood pressure, saturated oxygen with a finger oximeter and heart rate with a heart rate monitor. 


\section{Outcome Measure}

The following physiological parameters were retained: heart rate in bpm, saturation in the open air in\% was measured using a SANITAS oximeter, type SP025 / Germany. Respiratory rate in cycles per minute by inspection of respiratory movements and a stopwatch. The arterial pressure (BP) was measured, after 5 minutes of relaxation in a sitting position, with the left arm carried at the level of the heart using a mercury sphygmomanometer; three consecutive one-minute intervals were taken and their average defined the worker's AP. The PAS and PAD corresponded, respectively, to the I and $\mathrm{V}$ sounds of Korotkoff. HTA was defined as BP $\geq 140 / 90$ $\mathrm{mm} \mathrm{Hg}$ or as antihypertensive therapy regardless of PA (Kusuayi et al., 2018a). Respiratory volumes (FEV1, FVC and DEP) were measured using a Pocket Spirometer portable spirometer (Micro Medical Limited PO Box, 6 Rochester, Kent, ME 12 AZ, England). Cardio-vascular endurance and mobility were achieved by the six-minute walk test (TDM6) which consisted of walking for 6 minutes in a corridor (length 10 to 30 meters, depending on availability) as quickly as possible (Kusuay et al., 2018). The patient can stop walking at any time when he feels tired and resume walking. The important thing is to complete the required time (6 minutes). During the test, exercise HR, air saturation, perceived fatigue and dyspnea will be monitored by an HR monitor, a portable oximeter, and the Borg scale, respectively. The parameters analyzed after the test are the total distance traveled and the evolution of the heart rate using a cardio-frequency meter, an oximetry and a decameter, pads and a flat ground of 10 to 30 meters. The load (intensity) of the training was evaluated by the percentage of the maximum heart rate, expressed for the following equation: \% HR max $=$ (Exercise FC - Resting FC / HR max Resting FC) x 100 (Karvonen et al., 1983). Patients worked between $50 \%$ to $70 \%$ of the workload. This method relies on reserve $\mathrm{FC}=[(\%$ of desired exercise intensity * (HR max - FC rest) + rest FC] (Heyward, 2010) whose HR max in beat per minute (bpm) was evaluated at using the formula (Tanaka, 2001): HR $\max =208-(0.7 x$ age $)$. Dyspnea, fatigue and profuse perspiration were the subjective parameters of the upper limit of the tolerable load by the patients.

\section{Statistical analysis}

The collected data was captured and processed using the SPSS 21.0 software. Quantitative variables were expressed as means \pm standard deviation. The comparison of the averages of the continuous variables ( $\mathrm{T} 0$ versus $\mathrm{T} 3$ ) was performed by the paired Student's t-test. A value of $p \leq 0.05$ was considered as a threshold of statistical significance.

\section{Results}

\section{Table 1}

Compares physiological and clinical parameters related to physical performance before and after the program.

\begin{tabular}{lccc}
\hline Variables & Before & After & $\mathrm{p}$ \\
\hline FC 1 (bpm) & $99 \pm 6.2$ & $76 \pm 5.4$ & $<0.0001$ \\
FC 2 (bpm) & $155 \pm 10.1$ & $129 \pm 9.0$ & $<0.0001$ \\
PBS (mmHg) & $144 \pm 8.3$ & $130 \pm 4.2$ & $<0.0001$ \\
PBD (mmHg) & $100 \pm 7.0$ & $85 \pm 2.9$ & $<0.0001$ \\
FR (cycle/ min) & $28 \pm 1.7$ & $20 \pm 1.3$ & $<0.0001$ \\
DEP (liter/ second) & $6 \pm 1.9$ & $8.6 \pm 1.7$ & $<0.0001$ \\
CVF (liter/ second) & $3.124 \pm 0.8$ & $3.461 \pm 0.5$ & 0.0714 \\
FEV1 (liter/ second) & $1.968 \pm 0.7$ & $2.751 \pm 0.2$ & 0.0601 \\
TIFF (\%) & $62.9 \pm 14.0$ & $79.4 \pm 5.8$ & $<0.0001$ \\
Training load & $74.9 \pm 17.0$ & $54.2 \pm 12.2$ & $<0.0001$ \\
(\% FC max) & & & \\
$\begin{array}{l}\text { Dyspnea with effort } \\
\text { (min) }\end{array}$ & $10 \pm 4.7$ & $0 \pm 0.00$ & $<0.0001$ \\
$\begin{array}{l}\text { Fatigue with effort } \\
\text { (min) }\end{array}$ & $9 \pm 2.8$ & $60 \pm 1.6$ & $<0.0001$ \\
$\begin{array}{l}\text { SO } \text { in the open air } \\
\text { (\%) }\end{array}$ & $89 \pm 3.8$ & $95 \pm 4.2$ & $<0.0001$ \\
TDM Distance six & $100 \pm 17.9$ & $740 \pm 12.0$ & $<0.0001$ \\
(m) & & & \\
\hline
\end{tabular}

Maximum FC: 173.7 bpm: Beat per minute, FC1: Resting Heart Rate, FC2: Heart Rate immediately after exercise or exercise, PAS: Systolic Blood Pressure, PAD: Diastolic Blood Pressure, PEF: Expiratory Flow of Pointe, TIFF: Tiffeneau index, SO2: Oxygen saturation, $m m H g$ : millimeter of mercury min: minutes, m: meter.

Table 1 shows significant decrease of most parameters to study respectively: (- $23 \mathrm{bpm}) 99 \pm 6.2$ vs. $76 \pm 5,4 ; \mathrm{p}<0.0001$ for resting heart rate; $(-26 \mathrm{bpm})$ $155 \pm 10.1$ vs $129 \pm 9.0 ; \mathrm{p}<0.0001$ for heart rate immediately after exercise; $(-10 \mathrm{mmHg}) 144 \pm 8.3 \mathrm{vs}$ $130 \pm 4.2 ; \mathrm{p}<0.0001$ for systolic blood pressure; $(-8$ cycles per minute) $28 \pm 1.7$ vs $20 \pm 1.3$; $<<0.0001$ for the respiratory rate; $(-20.7 \%) 74.9 \pm 17.0$ vs $54.2 \pm 12.2$; $\mathrm{p}<0.0001$ for workload (intensity of effort) and (- 10 minutes) $10 \pm 4.7$ vs $0 \pm 0.00 ; p<0.0001$ for dyspnea on exertion. On the other hand, a significant increase of: (+ 2.6 liter per second) $6 \pm 1.9$ vs $8.6 \pm 1.7 ; \mathrm{p}<0.0001$ for Peak Expiratory Flow; (+ 19\%) $62.9 \pm 14.0$ vs 79.4 $\pm 5.8 ; \mathrm{p}<0.0001$ for the Tiffeneau index; (+51minutes) 
$9 \pm 2.8$ vs $60 \pm 1.6 ; \mathrm{p}<0.0001$ for the duration of sensation of fatigue during exercise; $(+6 \%) 89 \pm 3.8 \mathrm{vs}$ $95 \pm 4.2 ; \mathrm{p}<0.0001$ for oxygen saturation in the open air and ( +640 meters) $100 \pm 7.9$ vs $740 \pm 12.0 ; p<0.0001$ for the walking area.

\section{Discussion}

The present study showed, in 36 weeks of training in aerobic endurance and resistance, a significant decrease of most of the parameters to study respectively: resting heart rate $(-23 \mathrm{bpm})$; the heart rate immediately after exercise (- $26 \mathrm{bpm})$; systolic blood pressure $(-10 \mathrm{~mm} \mathrm{Hg})$; the respiratory rate $(-8$ cycles per minute); workload (intensity of effort) ($20.7 \%$ ) and dyspnea on exertion (- 10 minutes). In contrast, a significant increase in peak expiratory flow (+ 2.6 liter per second); the Tiffeneau index (+ $19 \%)$; the duration of sensation of fatigue at exercise (+ 51minutes); the oxygen saturation in the open air $(+6 \%)$ and the walking distance (+ 640 meters). In this regard, aerobic, resistance or combined exercise (resistance + aerobic) improves physical fitness in patients with COPD after the execution of training programs (Puhan et al., 2005; Vogiatzis et al., 2013). Many authors claim that aerobic endurance and resistance training improves workload by 15 to $30 \%$ and depends on the individual's level of fitness (Kust et al., 19977). This study also confirms this observation. Airway obstruction is detected when expired air in one second represents less than $75 \%$ of vital capacity (Tiffeneau Index less than 75\% (Pardy et al., 1976). Prior to training, an obstruction pulmonary pathways were detected in the patients and after training, we found a very significant improvement in the values of FEV 1, DEP, and Tiffeneau index. Ricci-Vitor et al. (2013) studying patients with in this regard, COPD detected an average difference in the distance of a six-minute walk test of $28.45 \mathrm{rn}$ after an 8-week resistance training program (24 sessions / h each). Our protocol differs from the protocol used by Ricci-Vitor et al. (2013), because they used static resistance of the lower and upper limbs with weights, while we used dynamic resistance with lining Similarly, similar results are presented in the Borghi-Silva et al. (2015), where they found a significant decrease in CF following the application of an aerobic exercise protocol for 12 weeks (36 sessions / $1 \mathrm{hr}$ each) also joining the decrease in vascular morbidity in patients with high fitness observed by Lokka et al. (1994) and Kusuayi et al. (2018b). Slight tachycardia and a tendency to maintain sympathovagal balance are characteristic in patients with COPD (Pagani et al., 1996). CF is a marker of sympathetic activity that is significantly higher in patients with COPD (van Gestel et al., 2012). Subsequent studies have demonstrated more convincingly that many patients with severely compromised pulmonary function may tolerate moderate high intensity endurance training and may achieve significant physiological gains in aerobic capacity (Rochester, 2003). ). A study by Mehri et al. (2007) in patients with COPD demonstrated that the completion of a pulmonary exercise program produces an increase in physical fitness, improves exercise tolerance, endurance of respiratory muscles, dyspnea, and quality of breathing life (Mehri, 2007). Cambach et al. (1999) performed a meta-analysis of clinical trials evaluating the long-term effects of training in patients with COPD or asthma. Studies show overall significant improvements in endurance exercise and maximum exercise capacity up to 9 months post exercise.

\section{Conclusion}

A training program for aerobic endurance and resistance significantly improves the stress tolerance of patients with Chronic Obstructive Pulmonary Disease and can be considered as an effective nonpharmacological management for the improvement of physical performance as well as that cardiorespiratory of these patients.

\section{References}

American Thoracic Society and European Respiratory Society. Skeletal muscle dysfunction in chronic obstructive pulmonary disease. a statement of the American Thoracic Society and European Respiratory Society. Am J Respir Crit Care Med, 1999; 159: S1 -40.

Borghi-Silva A, Mendes RG, Trimer R, Oliveira CR, Fregonezi GA, Resqueti VR, Costa D. Potential effect of 6 versus 12-weeks of physical training on cardiac autonomy function and exercise capacity in chronic obstructive pulmonary disease. Eur J Phys Rehabil Med, 2015; 51(2): 211-221.

Bryanton V, Chen Y, Johanson H, Leeb K, McRae A, Michealson P, Nair C, Stewart P. Respiratory disease in Canada Statistiques Canada, Santé Canada, 2001.

Cambach W, Wagenaar RC, Koelman TW, van Keimpema AR, Kemper HC. The long-term effects of pulmonary rehabilitation in patients with asthma and chronic obstructive pulmonary disease: a 
research synthesis. Arch Phys Med Rehabil, 1999; 80: 103-111.

Cambach W, Wagenaar RC, Koelman TW, van Keimpema AR, Kemper HC. The long-term effects of pulmonary rehabilitation in patients with asthma and chronic obstructive pulmonary disease: are search synthesis. Arch Phys Med Rehabil, 1999; 80(1): 103-111.

Camilli AE, Robbins DR, Lebowitz MD. Death certificate reporting of confirmed airways obstructive disease. Am J Epidemiol, 1991; 133: 795-800.

O’Donnell DE, Aaron S, Bourbeau J, Hemandez P, Marciniuk D, Balter M, Ford G, Gervais A, Goldstein R, Hodder R, Maltais F, Road J. Canadian Thoracic Society recommendations for management of chronic obstructive pulmonary disease-2003. Can Respir J, 2003; 10 Suppl A: 11A$65 \mathrm{~A}$.

Fahy BF. Pulmonary rehabilitation for chronic obstructive pulmonary disease: a scientific and political agenda. Respir Care, 2004; 49: 28-38.

Godefroid KM, Constant NE, Christophe D, Francois LB, Alosha NN. Effect of a structured physical exercises program on cardiovascular risk factors related to the metabolic syndrome in obese workers of Kinshasa in Democratic Republic of Congo. Sports and Science, 2018; 33(3): 115-122.

Godefroid KM, Constant NE, Christophe D, Francois LB, Alosha NN. Evaluation de la prevalence de hypertension artérielle de l'adulte en milieu du travail à Kinshasa, République Démocratique du Congo. Sports and Science, 2018; 33(4): 213-220.

Heyward V. Advanced fitness assessment and exercise prescription (Six th Edition ed.). US: Human Kinetics, 2010.

Jean-Jacques Gauthier, Philippe Bolduc, Yvon Cormier, and Pierre Nadeau. Pneumologie Clinique Les presses de l'Université de Montréal, 2002.

Karvonen J, Chwalbinska-moneta J, Pekkarinen H, Savola R. Abnormal ECG findings and heard function examined by non-invasive methods in a group of athletes. J Sports Med Phys Fitness, 1983; 23(4): 364372.

Kunsgea. Ventilatory muscle endurance training in normal subjects and patients with captic fibrosis. Am Rev Respir Dis, 1977; 41(4): 508-516.

Kusuayi MG, NKiama EC. Level of physical activity and eating behavior: Risk factors associated with sedentariness among employees of a company in the city of Kinshasa province. Turk J Kinesiol, 2018; 4(3): 82-90. DOI: 10.31459/turkjkin.439760

Kusuayi MG, Constant NE, Christophe D, Teddy BL, Nicias KN, Francois LB. Effects of a physical exercise program on the level of physical activity and energy expenditure of obese workers in Kinshasa in the Democratic Republic of Congo. International Journal of Kinesiology \& Sports Science, 2018; 6(2): 1- 6

Laçasse Y, Wong E, Guyatt GH, King D, Cook DJ, Goldstein RS. Meta-analysis of respiratory rehabilitation in chronic obstructive pulmonary disease. Lancet, 1996; 348: 1115-1119.

Lakka T, Vanalainen JM, Rauramaa R, Salomen R, Tuomiletho J. Relation of leisure-time physical activity and cardiorespiratory fitness to the risk of acute myocardial infarction. N Engl J Med, 1994; 330-22): 1549-1554.

Maltais F, Simard AA, Simard C, Jobin J, Desgagnes P, LeBlanc P. Oxidative capacity of the skeletal muscle and lactic acid kinetics during exercise in normal subjects and in patients with COPD. Am J Respir Crit Care Med, 1996; 153: 288-293.

Mehri SN, Khoshnevis MA, Zarrehbinan F, Hafezi S, Ghasemi A, Ebadi A. Effect of treadmill exercise training on $\mathrm{VO}_{2}$ peak in chronic obstructive pulmonary Disease. Tanaffos, 2007; 6(4): 18-24.

National Center for Health Statistics. Death: final data for 2001. National Vital Statistics Report 52, 2003.

Pagani M, Lucini D, Pizzinelli P, Sergi M, Bosisio E, Mela GS, Malliani A. Effects of aging and of chronic obstructive pulmonarydisease on RR intervalvariability. J Auton Nerv Syst, 1996; 59(3): 125-132.

Pardy R, Rivington R, Despas P, Macklem P. Inspiratory muscle training compared with physiotherapy in pateints with chronic airflow limitation. Am Rev Respir Dis, 1981; 123(4): 421-425.

Pauwels RA, Buist AS, Calverley PM, Jenkins CR, Hurd SS, Gold Scientific Committee. Global strategy for the diagnosis, management, and prévention of chronic obstructive pulmonary disease. NHLBI/WHO global initiative for chronic obstructive lung disease (GOLD) workshop summary. 2001; Am J Respir Crit Care Med, 163: 1256-1276.

Puhan M, Schunemann H, Frey M, Scharplatz M, Bachmann L. How should COPD patients exercise during respiratory rehabilitation? Comparison of exercise modalities and intensities to treat skeletal muscle dysfunction. Thorax, 2005; 60(5): 367-375. doi: 10.1136/thx.2004.033274

Ricci-Vitor AL, Bonfim R, Fosco LC, Bertolini GN, Ramos EM, Ramos D, Pastre CM, Godoy M, Vanderlei LC. Influence of the resistance training on heart rate variability, functional capacity and muscle strength in the chronic obstructive pulmonary disease. Eur J Phys Rehabil Med, 2013; 49(6): 793-801. 
Ries AL, Carlin BW, Carrieri-Kohlman V, Casaburi R, Celli BR, Emery CF, Hodgkin JE, Mahler DA, Make B, Skolnick J. Pulmonary Rehabilitation: Joint ACCP/AACVPR Evidence-Based Guidelines. J Cardiopulm Rehabil, 1997; 17(6): 371-405.

Rochester CL. Exercise training in chronic obstructive pulmonary disease. J Rehabil Res Dev, 2003; 40(5 Suppl 2): 59-80.

Salman GF, Mosier MC, Beasley BW, Calkins DR. Rehabilitation for patients with chronic obstructive pulmonary disease: meta- analysis of randomized controlled trials. J Gen Intern Med, 2003; 18: 213221.

Tomas LH, Varkey B. Improving health-related quality of life in chronic obstructive pulmonary disease. Curr Opin Pulm Med, 2004; 10: 120-127.
Van Gestel AJ, Kohler M, Steier J, Sommerwerck U, Teschler S, Russi EW, Teschler H. Cardiac autonomie function and cardiovascular response to exercise in patients with chronic obstructive pulmonary disease. Int J Chron Obstruct Pulmon Dis, 2012; 9(2): 160-165. doi: 10.3109115412555.2011.647130

Vogiatzis I, Zakynthinos S. The physiological basis of rehabilitation in chronic heart and lung disease. J Appl Physiol (1985), 2013; 115(1): 16-21. doi: 10.1152/japplphysiol.OO 195.2013

Walk JA, Hart CL, Soriano JB. What do COPD patients die of? A multiple cause coding analysis for England and Wales from 1993-99. Am J Respir Crit Care Med, 2003; 167(7): A237. 